\title{
ECOLOGY OF PARASITES
}

$\mathrm{T}$ HE autumn meeting of the Parasitology Group (Institute of Biology) was held in London at Birkbeck College on October 30, 1959. In the opening discussion of the meeting, which was entitled "The Ecology of Parasites", Prof. B. G. Peters (Imperial College, London) considered the effect of soil factors on plant parasitic nematodes. Soil-living stages are directly influenced by edaphic factors such as moisture, temperature, chemical constitution of the soil and the presence of predators and of possible hosts. Prof. Peters went on to point out that in the parasitic phases, complex relationships exist between the biomasses of host plants and parasitic eelworms. With increasing plant nutrients in the soil there is an increase in host biomass and a correlated increase in parasite biomass. However, at a fixed nutrient-level, the correlation between host and parasite biomasses is negative and the parasites retard the development of their hosts. In species of Heterodera having one generation each season, the multiplication rate is highest at low population-levels. As the parasite population density builds up so the rate of multiplication falls. Concurrently, the host at a fixed population density shows a correlated fall in biomass until it can barely maintain the eelworm population. Under these conditions, catastrophic crop losses occur in one season and can alternate with reasonable crop yields the next.

Dr. R. Lainson (London School of Hygiene and Tropical Medicine) spoke about the adaptations shown by coccidial parasites to their environment. The life-histories of sporozoan Protozoa show striking modifications which may be regarded as allowing the parasite to overcome the hazards of transmission and which form an evolutionary pattern, often serving as a basis of classification.

Eimeria, a typical coccidian, has one host in its life-cycle and the difficulties of transfer are overcome by the production of resistant oocysts, which can remain viable outside the host for long periods. The hæmogregarines have eliminated the dangers of a free-living stage and are passively transferred by octoparasitic invertebrates such as leeches, ticks and mites. The parasites circulating in the blood cells of the vertebrate are ingested by a blood-feeding invertebrate, which itself is eaten by a new vertebrate host.

Yet other coccidians are further adapted and undergo most of their life-cycle in the ectoparasite, the vertebrate virtually serving as a reservoir of infection for the invertebrate host. Besides these modifications in the mechanism of transmission, changes have taken place in the processes of fertilization and sporogony, and all these adaptations ensure maximum efficiency in the maintenance and dissemination of the species.

Dr. S. D. Garrett (Botany School, Cambridge) discussed inoeulum potential. Infectivity denotes the potentiality for infection of a single infective organism. In order to produce disease in 50 per cent of inoculated host individuals a large dose of infective agents is often required, and the term "inoculum potential' denotes the infection potential of this dose. The necessity for the large dose in establishing infection can be explained either by the low probability for infection by a single organism, the hypothesis of independent action, or by the necessity for synergic action between individual infective organisms - the hypothesis of synergistic action.

Evidence available from plant pathology supports that from animal pathogens and accords better with the hypothesis of independent action. However, the possibility still exists that synergism may be involved in the production of disease in an 'unnatural' host, for example, where it is necessary to apply heavy doses of rust fungus spores to the leaf surface to produce disease. The chief evidence of synergistic action is provided by experiments with fungi infecting the roots of trees. The high resistance of the roots can only be overcome by a fungal inoculum provided with a food base, and infection is accomplished by mycelial strands and not by individual hyphæ.

Dr. G. Salt (King's College, Cambridge) spoke about the environment of larval parasitoid insects. The ecology of these parasitoids has two distinct aspects. The adults are free-living, usually winged, and the ways in which the females select the hosts and parasitize them have been widely studied. How. ever, the ecology of the parasitoid egg or larva living on or in a particular host has received relatively little attention. Dr. Salt described some of his work on the life-history of a parasitoid larva within the hrmocol of its larval insect host and gave an account of the environment, the changes which must occur in it if the parasite is to survive and the interactions between host and larva. An 'unnatural' host reacts to the presence of the larva in its hæmocœel, the larva becoming surrounded by an envelope of host blood which prevents development.

Dr. D. Arthur (King's College, London) described the ways in which ticks select their hosts and the feeding sites upon them. Hard ticks, on the tips of vegetation, are aroused to a state of preparedness by non-directional, tactile stimuli. These stimuli are reinforced by interacting odour and temperature stimuli prior to attachment to the host. In soft, ticks, temperature appears to be the dominant stimulus for attachment.

The range of hosts selected by the parasite is not precisely known because of incorrect identification of ticks by earlier workers. However, the majority of ticks appear to be non-specific in their host selection. Some are limited to certain groups of hosts, but very few are specific to one host species, even among primitive ticks feeding on primitive mammals and the more advanced forms such as those found exclusively on Primates. For example, Ornithodorus moubata appears in three biological forms, that found on warthogs, porcupines and antbears possibly being primitive. The sites of attachment of various species and the reasons for their selection were discussed.

The chairman, Dr. E. L. Taylor (Veterinary Laboratory, Weybridge), opened the discussion. The adaptations of a parasite show various aspects 


\title{
No. 4709 January $30,1960 \quad$ N A T U R E
}

operating simultaneously. The ecology of stages outside the host is similar to that for free-living organisms, but the parasite has also to find its host, effect an entrance or attachment and then behave in a way that ensures its own and its host's continued existence. The effect of parasite on host has been widely studied, but the effect of the host on the parasite should also be considered. Indeed, the two physiologies are interacting and in some circumstances mutual benefit may accrue. Contrary to the usual tenet of successful parasitism, the parasitoid insect larva kills its host, and Dr. Salt commented that the percentage of host population infected was small and losses, which could be considered as due to a refined form of predation, could easily be supported. Dr. Hindle cited examples of the extinction of host species by parasitism. Immunity reactions of the host, although prejudicial to an individual parasite, operate for the benefit of the species by limiting the parasite population. This mechanism is different in plant and animal hosts, and Dr. Jones said that plants do not show immune reactions, but rather a resistance to infestation which is a property of the individual cells. Parasite populations of plants show both density. dependent effects and independent factors, such as the steady rate of decay of the population of cystforming eelworms in the soil.

Immunity in invertebrates has been little studied, but there appears to be no true immunity although the production of non-specific antibodies may be evoked by the introduction of any foreign object. When the parasitoid insect larva with its envelope of host blood is transferred to another similar host there is no homograft reaction such as occurs in vertebrates.

Dr. Garrett suggested that another important difference between the plant and animal host is that in the plant there is always a region of young tissue at the growing points, whereas in animals growth usually ceases at maturity. Young animals are more susceptible than adults, and in plants the rootlets, for example, are more susceptible than the roots, the resistance of which can only be overcome by the most specialized of parasites. This phenomenon of ageresistance also operates in favour of the parasite by limiting the population.

The proceedings of this meeting will not be published in full.
JUNE MahoN

\section{ORIGIN OF TEKTITES}

\author{
BY DR. GEORGE BAKER \\ Commonwealth Scientific and Industrial Research Organization, Mineragraphic Investigations, \\ c/o University of Melbourne, Parkville, N.2, Victoria
}

\begin{abstract}
CERTAIN aspects of recently advanced concepts 4 relating to the origin of tektites require some considerable modification in the light of the conclusions arising from long-continued and detailed studies of the tektites from Australia. So far as is known, australites are the most recently arrived tektites on the Earth, and have evidently not lain upon the Earth's surface for more than 5,000 years. Furthermore, in terms of preservation of original shape types modified by secondary phenomena generated during rapid transit through the Earth's atmosphere, they are unmatched by any tektites from any other strewn-field on the Earth. Consequently, they are in the best condition on which to base conclusions as to their origin. Moreover, the very best preserved, most perfectly developed australites, which come from the Port Campbell district on the southern coast of Western Victoria, are retained in Australia, and have not been critically examined by overseas writers who make reference to australites in their publications.
\end{abstract}

Much detailed information has now accrued relating to the primary shapes, the secondarily modified shapes arising from the action of aerodynamical phenomena during ultra-supersonic and supersonic flight through the Earth's atmosphere, and the effects of tertiary phenomena such as subaerial erosion resulting from 5,000 years exposure to atmospheric agents. Considerable and detailed studies of the specific gravity of the same and different types of shape from the same and from different localities have been carried out. Many refractive index determinations have been made and specific refractivities calculated. Arcs and radii of curvature of remnant primary (posterior) and newly formed secondary (anterior) surfaces have been determined graphically from silhouette tracings, and significant conclusions drawn from these. Several etch solution tests have been conducted which provide valuable information of the effects of natural etching upon original structural elements and sculpture patterns of australites. Three more chemical analyses have been made and the composition related to specific gravity and refractive index values. The effects of heating to $1,200^{\circ} \mathrm{C}$. has added further knowledge to the australite problem generally.

Studies of Darwin glass, 'straw-silica glass' and other non-volcanic terrestrial glasses have led to the rejection of Darwin glass from the group of the true tektites. Thin-section studies of the internal schlieren of australites have clarified the relationships between the core or body portion and the circumferential flange. All the structures, sculpture patterns and present shapes of australites can be adequately explained in terms of the few primary forms developed in an extraterrestrial environment, and subsequent modification by aerodynamical phenomena during high-speed earthward flight through the Earth's atmosphere, with a further modification by fragmentation, abrasion and solution-etching of the shapes so developed.

The results of these and other detailed studies have appeared during the past two decades ${ }^{1}$.

The question of the origin of australites is admittedly still unsolved, but the weight of evidence from Australian studies is strongly in favour of an extraterrestrial source.

The concept has recently been advanced ${ }^{2}$ that the arrival of tektites from space, with the distribution found on Earth, is not possible. Urey has calculated 